\section{Rôle de la}

fractalkine/

CX3CL1 et de

son récepteur

CX3CR1 dans

les pathologies

allergiques

Valérie Julia ${ }^{1}$, Delphine Staumont-Salle ${ }^{2}$, David Dombrowicz ${ }^{2}$

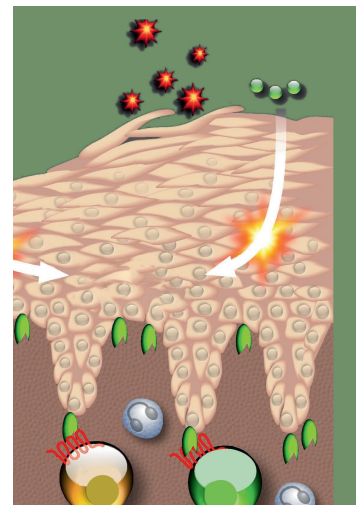

${ }^{1}$ CNRS, Inserm, université de Nice-Sophia Antipolis, institut de pharmacologie moléculaire et cellulaire, 660 , route des Lucioles, 06560 Valbonne, France ;

${ }^{2}$ Université de Lille, Inserm, CHU Lille, European genomic institute of diabetes, institut Pasteur de Lille, U1011 récepteurs nucléaires, maladies cardiovasculaires et diabète, 59000 Lille, France. vjulia@ipmc.cnrs.fr david.dombrowicz@pasteur-lille.fr

Les maladies atopiques affectent jusqu'à $30 \%$ de la population et leur prévalence a très fortement augmenté au cours des dernières décennies dans les pays industrialisés en raison des changements intervenant non seulement dans l'environnement mais touchant également le mode de vie [1]. Ces pathologies diffèrent par leur organe cible mais elles partagent une étiologie commune, ce qui les fait considérer comme un continuum physiopathologique: «la marche atopique ». La dermatite atopique (DA) est une pathologie cutanée inflammatoire chronique avec rechutes récurrentes. Elle survient généralement au cours de la petite enfance mais peut persister ou même débuter à l'âge adulte. La DA résulte d'interactions complexes entre des gènes de susceptibilité, l'environnement, un défaut de la barrière cutanée et des réponses immunologiques. On considère qu'il s'agit de l'expression cutanée d'un désordre systémique qui conduit également au développement de l'asthme, de la conjonctivite et de la rhinite allergiques. Au moins deux formes de DA ont été décrites: la dermatite atopique extrinsèque correspondant à la dermatite atopique allergique ${ }^{1}$ et la dermatite atopique intrinsèque ${ }^{2}$. La DA extrinsèque touche 70-80\% des patients et est associée à une sensibilisation allergénique dépendant des $\lg \varepsilon$. Dans les pays

\footnotetext{
${ }^{1}$ La nouvelle nomenclature internationale allergologique conseille d'appeler cette dermatite atopique extrinsèque « eczéma atopique».

${ }^{2}$ La dermatite atopique intrinsèque, que la nouvelle nomenclature suggère d'appeler « eczéma », correspond à une DA sans aucun terrain atopique associé.
} 
industrialisés, la prévalence de la DA est de 10-20\% chez les enfants et de 1-3\% chez les adultes [2]. Plus de $50 \%$ des enfants atteints de DA développent ultérieurement une pathologie asthmatique et $75 \%$ souffrent de rhinite allergique avant l'âge de 6 ans [3]. La prévalence de la DA a augmenté d'environ 2 à 3 fois au cours des trois dernières décennies. Dans ses formes sévères, la DA peut être extrêmement invalidante et sa prise en charge est complexe. L'asthme allergique [48] est une pathologie pulmonaire chronique caractérisée par une hyperréactivité bronchique (HRB), une production excessive de mucus, une éosinophilie pulmonaire ${ }^{3}$ et un remodelage des voies aériennes [4]. Les individus affectés présentent une morbidité significative. Au niveau mondial, l'asthme affecte actuellement 300 millions d'individus [5]. Comme dans le cas de la dermatite atopique, la prévalence de l'asthme a significativement augmenté au cours des dernières décennies. Chez l'homme et la souris, l'inflammation allergique résulte principalement de l'activation des lymphocytes $T$ auxiliaires de type Th2 qui produisent des cytokines favorisant la production d'Ig $\varepsilon$ et l'éosinophilie. Le recrutement et la persistance des lymphocytes Th2 dans les voies aériennes et la peau sont des événements essentiels dans la pathogenèse de l'asthme et de la DA. Alors que le rôle des chimiokines dans le recrutement des lymphocytes T est largement étudié, très peu d'études sont consacrées à leur implication dans la survie cellulaire. Cet aspect a très probablement été négligé en partie parce qu'il peut être confondu avec le processus de migration cellulaire, les deux phénomènes conduisant à des résultats similaires au sein du tissu. L'absence ou la neutralisation des chimiokines peut altérer l'accumulation des cellules au sein du tissu inflammatoire en faisant intervenir d'autres paramètres que le recrutement. $\varepsilon$ n effet, une réduction de l'accumulation cellulaire peut être le résultat d'une altération de la survie ou de la rétention des cellules au sein du tissu d'intérêt. Dans la plupart des cas, le mécanisme d'action des chimiokines et la spécificité cellulaire de certains de leurs récepteurs demeurent mal connus.

\section{La fractalkine}

CX3CLl (chemokine [C-X3-C motif] ligand 1), également appelée fractalkine ou neurotactine, est une protéine transmembranaire de type $I^{4}$ composée d'un domaine chimiokine CX3C situé à l'extrémité longue séquence glycosylée de la molécule [6]. À ce jour, seules CX3CLl et CXCL16, le ligand de CXCR6 (chemokine [C-X-C motif] receptor 6 ), ont été décrites pour présenter un ancrage membranaire. CX3CL1 est clivée de la surface cellulaire par ADAM-10 (A disintegrin and metalloproteinase domain-containing protein 10) ou par TACE (tumor-necrosis factor-[TNF]- $\alpha$-converting enzyme, ou ADAM-17) [6]. L'activité de TACE est induite in vitro par la stimulation de cellules épithéliales pulmonaires humaines par l'IL(interleukine)-13 [7]. Dans le modèle de poumons inflammatoires chez la souris, TACE est activée à la suite d'une sensibilisation et une épreuve d'ovalbumine

${ }^{3}$ Infiltrat d'éosinophiles au niveau pulmonaire. L'éosinophile est souvent associé à des pathologies allergique ou parasitaire. Une éosinophilie supérieure à $2 \%$ des leucocytes dans le liquide bronchoalvéolaire est considérée comme anormale.

${ }^{4}$ Constituée d'une chaîne polypeptidique qui traverse une fois la double couche lipidique.
(OVA) utilisée comme allergène [8]. CX3CL1 se lie à un seul récepteur, $C X 3 C R 1$. Chez l'homme, $C X 3 C R 1$ possède deux ligands connus: CX3CL1 et CCL26 (chemokine [C-C motif] ligand 26, ou eotaxin-3) [9] mais chez la souris, le gène codant CCL26 étant un pseudogène (non fonctionnel), le récepteur ne compte que CX3CLl comme ligand connu.

\section{Distribution cellulaire de CX3CL1}

Chez l'homme, CX3CLl est exprimée par divers types cellulaires et tissus dont les neurones [10], l'épithélium intestinal [11] et pulmonaire [12], ainsi que la peau [13], les cellules musculaires lisses pulmonaires [14] et l'endothélium ombilical [6]. Chez la souris, les principales données sur la localisation de $\mathrm{CX} 3 \mathrm{CL}$ l proviennent de la génération d'une lignée de souris exprimant une protéine rapporteur fluorescente comme marqueur de substitution pour l'expression de la chimiokine [15]. Elles confirment les observations obtenues chez l'homme et précisent, au sein des tissus, les types cellulaires qui expriment CX3CL1 : les cellules caliciformes intestinales, l'épithélium alvéolaire et bronchique et les cellules de Clara épithéliales ${ }^{5}$, les cellules épithéliales des tubules et des glomérules. En revanche, l'expression de CX3CLI n'a pas été détectée sur l'endothélium vasculaire de souris naïves.

\section{Distribution cellulaire du récepteur CX3CR1 en conditions homéostatiques}

L'expression du récepteur de CX3CL1, CX3CR1, chez I'homme, peut être aisément étudiée en cytométrie de flux et par immmunohistochimie à l'aide d'anticorps (disponibles dans le commerce). Ainsi, CX3CRl est retrouvé dans les neurones [16], les cellules microgliales [16], les monocytes [17], les cellules natural killer $\mathrm{CD}_{56}{ }^{\mathrm{dim}} \mathrm{CD} 16^{+}$(NK) et des sous-populations de lymphocytes T [17]. Chez la souris, aucun réactif spécifique de CX3CRI n'étant disponible, l'expression du récepteur a été étudiée à l'aide d'animaux hémizygotes pour lesquels un allèle de CX3CRI a été remplacé par le gène codant la protéine fluorescente eGFP (enhanced green fluorescent protein) afin de pouvoir le tracer [18]. Comme chez l'homme, CX3CRl est fortement exprimé dans les cellules microgliales murines. II est également exprimé sur les monocytes sanguins, divers types de progéniteurs de cellules dendritiques (CD)

\footnotetext{
${ }^{5}$ Les cellules de Clara sont des cellules de l'épithélium des bronchioles terminales. Ce sont des cellules pyramidales dont le pôle apical est garni de microvillosités. Dans la partie apicale de leur cytoplasme existent des grains de sécrétion (compo-
} sés d'antiprotéases et d'oxydases) expulsés par exocytose. 


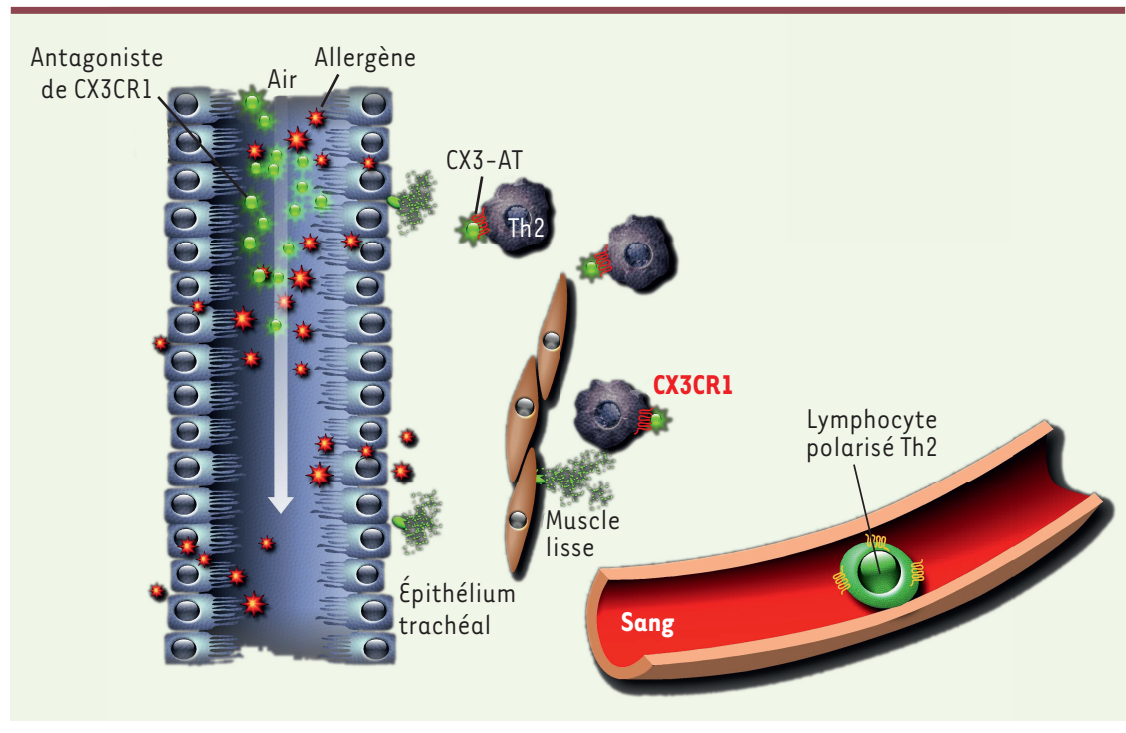

Figure 1. Fractalkine dans le poumon allergique. Les lymphocytes T spécifiques de l'allergène migrent dans les poumons indépendamment de CX3CRI (CX3C chemokine receptor 1 ) puis expriment ce récepteur une fois dans le tissu. L'interaction avec la fractalkine exprimée par les cellules bronchiques épithéliales ou par les cellules du muscle lisse permet la survie des ces derniers au sein du tissu pulmonaire inflammatoire. L'administration locale d'un antagoniste peptidique de CX3CL] (chemokine [C-X3-C motif] ligand 1) bloque le signal de survie induit par la fractalkine, prévient l'hyperréactivité bronchique et l'inflammation pulmonaire chez la souris. CX3-AT : antagoniste de CX3CRI.

[19], les CD plasmacytoïdes $(p C D)^{6}$, les cellules NK, les macrophages de la lamina propria ${ }^{7}$ et les lymphocytes $B$ intestinaux tolérogéniques [20] et T CD8 ${ }^{+}[18]$.

\section{L'axe CX3CL1-CX3CR1 dans les pathologies allergiques}

Un rôle pour la chimiokine $C X 3 C L 1$ et son récepteur CX3CRl a été suspecté dans le contexte de nombreuses maladies inflammatoires au cours desquelles l'expression de CX3CLI est élevée comme le psoriasis $[21,49](\rightarrow)$, la bronchopneumopathie obstructive [22], l'hypertension [23] et la fibrose pulmonaire [24]. Il a été par ailleurs proposé que CX3CRI participait à la pathogenèse de ces affections en favorisant la transmigration des monocytes et/ou des $\rightarrow$ Voir la Synthèse de A. de Masson et al., page 253 de ce numéro lymphocytes exprimant le récepteur. Par contre, le rôle de CX3CRI a été clairement établi dans l'arthrite rhumatoïde (voir [25] pour une revue) et dans l'athérosclérose [26].

De nombreux résultats suggèrent que les interactions établies entre CX3CLl et CX3CR1 jouent un rôle important dans les pathologies allergiques. Ainsi, des analyses génétiques ont montré que deux polymorphismes nucléotidiques (SNP, single-nucleotide polymorphism) de CX3CRI sont respectivement associés à l'asthme et à l'atopie dans des populations franco-canadiennes [27] et une cohorte d'enfants alle-

${ }^{6}$ Les CD plasmocytoïdes (anciennement appelées CD2 car elles orientent la réponse immunitaire vers la production de cytokines de type 2 : IL-4 et IL-5).

${ }^{7}$ La lamina propria est située sous les épithéliums des muqueuses digestives, respiratoires ou urogénitales. mands [28]. De même, des concentrations élevées de $\mathrm{CX} 3 \mathrm{CL}$ l dans le sérum et le lavage bronchoalvéolaire (BALF) sont retrouvées chez les patients atteints de rhinite ou d'asthme allergiques. Cellesci augmentent encore après un test d'épreuve à l'allergène chez les patients allergiques asthmatiques [12]. Chez les patients asthmatiques, l'expression de la chimiokine est également augmentée dans les cellules musculaires lisses des voies aériennes, l'endothélium pulmonaire et l'épithélium bronchique suite à un test d'épreuve [14]. Dans leur ensemble, ces résultats montrent que, chez les patients allergiques, $\mathrm{C} \times 3 \mathrm{CLI}$ est activé par clivage, vraisemblablement à la suite de l'activation d'ADAM17 (qui initie la réaction de coupure de la chimiokine). De plus, chez les patients atteints de rhinite ou d'asthme allergiques, une proportion de lymphocytes $T$ $\mathrm{CD}^{+}{ }^{+}$effecteurs/mémoires qui expriment CX3CRI est retrouvée et une activation de la signalisation de CX3CRI en réponse à CX3CL1 est observée, ce qui n'est pas le cas chez les individus sains [12]. En accord avec ces observations suggérant le rôle critique des interactions CX3CL1CX3CR1 dans les pathologies allergiques, nous avons montré dans un modèle murin d'asthme, que les souris déficientes en CX3CRI, ou des souris de type sauvage (WT) traitées avec un antagoniste de CX3CR1, présentent des symptômes asthmatiques fortement réduits [29]. De plus, le transfert de lymphocytes T CD4 $4^{+}$des souris sauvages dans des animaux déficients en CX3CRI restaure l'amplitude de la réaction asthmatique. De même, les antagonistes de CX3CRI inhibent l'asthme induit par le transfert de lymphocytes de souris sauvages polarisés Th2 chez des animaux déficients en CX3CR1 (Figure 1). Ces résultats montrent ainsi que l'expression de CX3CRI par les lymphocytes $T \mathrm{CD} 4^{+}$effecteurs est nécessaire au développement de l'asthme.

Dans la DA, les données cliniques quant au rôle des interactions CX3CL1-CX3CRI sont beaucoup plus parcellaires. Chez les patients avec DA, l'expression de la chimiokine CX3CLl est augmentée à la fois sur l'endothélium et au niveau des lésions cutanées [13]. Les concentrations sériques de la chimiokine sont associées positivement à la sévérité de la pathologie et inversement corrélées à l'amélioration clinique des lésions. Une étude récente a montré que les niveaux d'ARN messagers (ARNm) spécifiques du récepteur CX3CRl sont augmentés dans 


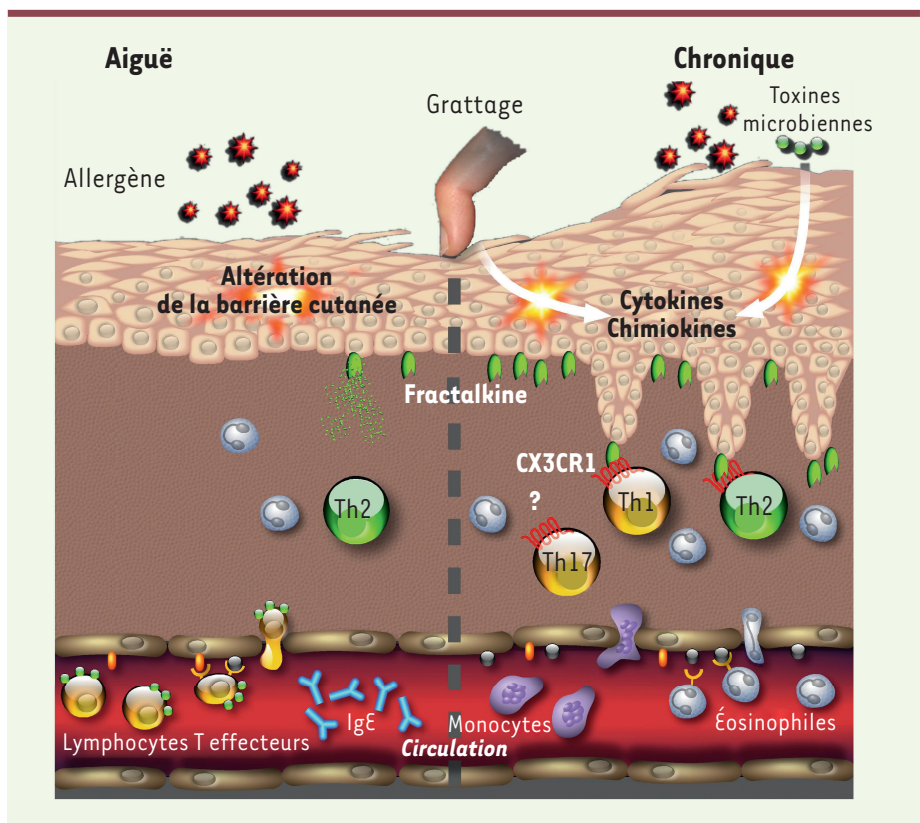

Figure 2. Fractalkine dans la peau allergique. Alors que la migration des lymphocytes T spécifiques de l'allergène dans la peau ne requiert pas CX3CRI (CX3C chemokine receptor 1 ), une fois dans le tissu inflammatoire cutané, les lymphocytes Th2 et Th1 expriment le récepteur CX3CR1. L'interaction avec son unique ligand i.e. fractalkine ( $\mathrm{CX} 3 \mathrm{CL}$ l chemokine [C-X3-C motif] ligand I) exprimée principalement par les cellules épithéliales permet la rétention de ces derniers dans le tissu. II reste à déterminer si les autres acteurs de la dermatite atopique tels que les Th17 et Th22 utilisent aussi CX3CR1 comme récepteur d'« ancrage » dans le tissu cutané inflammatoire.

la peau des patients avec DA. Cependant, le taux d'ARNm spécifique de CX3CL1 n'est accru que chez certains patients, avec une corrélation significative avec la sévérité de la maladie [9]. Ces résultats pourraient expliquer l'absence de détection de l'expression de la chimiokine CX3CLI dans la peau de quatre patients avec DA [21]. Chez la souris, comme pour l'asthme allergique, nous avons montré dans un modèle de DA très proche de la pathologie humaine, en utilisant des souris déficientes en CX3CR1 et des réactifs bloquant les interactions CX3CR1-CX3CL1, que ces interactions contribuaient à la pathologie de manière plus importante que dans le cas de l'asthme [30] (Figure 2). Cette différence peut être expliquée par le fait que, plus que l'asthme allergique, la dermatite atopique - et le modèle expérimental utilisé - est fortement dépendante, dans sa phase chronique, de la réponse des lymphocytes Thl qui expriment des niveaux élevés de CX3CRI (résultats non publiés). En ce qui concerne les autres pathologies cutanées, malgré l'expression accrue de CX3CLI rapportée dans le cas du psoriasis, nous n'avons pas détecté d'augmentation significative de l'expression du récepteur CX3CRI sur des lymphocytes cutanés chez l'homme. De même, dans le modèle murin, les souris déficientes en CX3CRI développent une pathologie psoriasique expérimentale (et une réaction d'hypersensibilité de contact) qui est similaire à celle observée pour des souris sauvages [30]. En revanche, chez les patients avec DA, nous avons mis en évidence l'expression de CX3CRl sur les lymphocytes T CD4 $4^{+}$infiltrant les lésions cutanées et exprimant des cytokines Th1 ou Th2 [30]. Ces résultats suggèrent donc que, bien que les contributions de l'expression de CX3CRI selon les différents types de lymphocytes soient distinctes de celles observées dans l'asthme, ce récepteur joue un rôle important dans une pathologie allergique cutanée alors qu'il ne semble pas intervenir dans d'autres pathologies inflammatoires cutanées.

\section{Régulation de l'expression du récepteur CX3CR1}

Si une sous-population de lymphocytes T CD $4^{+}$effecteurs/ mémoires exprime le récepteur $C X 3 C R 1$ chez des patients asthmatiques [12], leur phénotype n'a actuellement pas été complètement caractérisé. Ils expriment le récepteur aux chimiokines CCR5 (chemokine [C-C motif] receptor 5) pouvant suggérer un phénotype Thl pour ces lymphocytes [31]. Similairement, des lymphocytes $T$ isolés du sang de cordon ombilical et polarisés in vitro vers un profil Thl, présentent des niveaux élevés d'ARNm spécifique de CX3CRI alors que ce n'est pas le cas pour des cellules ayant été polarisées en Th2 [21]. Chez la souris, nous avons montré que les lymphocytes Thl et Th2, polarisés in vitro en présence de splénocytes et d'antigène, n'expriment pas CX3CR1, à moins d'être incubés avec des cellules pulmonaires, ou d'être injectés à des animaux exposés par aérosol ou par voie épicutanée (sur la peau intacte) à leur antigène spécifique. Ces résultats suggèrent ainsi l'existence d'une fine régulation de l'expression de CX3CRI. Chez l'homme, l'expression du gène codant le récepteur est contrôlée par trois régions promotrices distinctes ce qui conduit à la transcription de trois ARNm matures qui diffèrent seulement au niveau de leur extrémité non traduite [32]. Cette régulation complexe au niveau génique permet vraisemblablement une expression hautement variable en fonction du type cellulaire et du microenvironnement. Bien que la régulation ait été peu étudiée dans les cellules hématopoḯtiques, il a été montré que chez l'homme, l'IL-2 induisait l'expression de CX3CRI sur les lymphocytes T CD4 et CD8 [17], et que l'IL-10 et I'IFN- $\gamma$ augmentaient cette expression dans des lignées de monocytes [33]. Dans les cellules NK humaines CD56 ${ }^{+}$, l'expression du récepteur est également augmentée par I'IL-2 mais elle est inhibée par l'IL-15 [34]. Les cytokines/molécules responsables de l'expression de CX3CR1 sur les lymphocytes $\mathrm{TCD}^{+}$dans le poumon ou la peau des souris allergiques restent à déterminer.

\section{Migration, survie ou rétention?}

Bien que les chimiokines aient été originellement définies comme des chimioattractants permettant le recrutement des cellules, un nombre croissant de données suggèrent 
qu'elles favorisent également la survie cellulaire. Le rôle antiaptoptotique de CX3CLl a d'abord été identifié dans les neurones et la microglie. En effet, CX3CLl réduit l'apoptose neuronale induite par la protéine d'enveloppe gp120 du VIH-1 (virus de l'immunodéficience humaine de type 1), un effet qui est bloqué par des anticorps spécifiques de CX3CRl [35]. De même, CX3CL1 réduit l'apoptose neuronale induite par CPAF(HIV-I neurotoxin platelet activating factor) ou la protéine régulatrice du virus, Tat, confirmant l'effet neuroprotecteur de CX3CLI au cours de l'infection par le VIH. CX3CLI inhibe également l'apoptose de la microglie induite par le récepteur proapoptotique Fas [37] ou par des lipopolysaccharides bactériens (LPS) [38]. Ces différentes études suggèrent donc que les interactions entre CX3CLl et son récepteur CX3CRI promeuvent la survie neuronale et microgliale, au moins dans des conditions pathologiques. Ces interactions interviennent également dans la survie des monocytes, notamment en conditions homéostatiques et au cours de l'athérosclérose [39]. Si cet effet est médié par la forme transmembranaire de CX3CL1 [15], la forme soluble de la chimiokine inhibe également la mort des monocytes humains carencés en sérum in vitro [39]. Les interactions de la chimiokine avec son récepteur favorisent la survie lymphocytaire au site inflammatoire. En effet, une survie des lymphocytes, impliquant CX3CR1, a été observée pour les lymphocytes Thl et pour les lymphocytes Th2 au cours de l'inflammation pulmonaire, mais pas en conditions homéostatiques, ni dans des ganglions lymphatiques inflammatoires [29]. Cela conforte l'observation que les lymphocytes $\mathrm{T}_{\mathrm{CD}} 4^{+}$effecteurs n'expriment pas CX3CRl en périphérie mais exclusivement dans les tissus inflammatoires. Comme dans le cas des monocytes, la survie des lymphocytes Th2 peut être induite in vitro par la forme soluble de CX3CL1. Au niveau cutané, les interactions CX3CL1CX3CRl ne conditionnent ni la migration, ni la prolifération, ni la survie des lymphocytes présents au site inflammatoire (qui sont comparables, que les lymphocytes expriment ou non $(X 3 C R 1$ ), mais régulent la rétention des lymphocytes Thl et Th2 au niveau du site, définissant ainsi un rôle nouveau pour une chimiokine [30]. À l'heure actuelle, l'origine de cette nouvelle contribution des interactions CX3CLI-CX3CRI au niveau lymphocytaire ne peut être explorée et démontrée. Nous pouvons néanmoins supposer que les cellules qui expriment la chimiokine CX3CLl, requise pour la signalisation lymphocytaire via son récepteur CX3CRl, sont différentes au niveau pulmonaire et cutané. Alors que dans la peau, CX3CLl est exprimée par les cellules épithéliales et endothéliales et au niveau des lésions présentées par les patients atopiques, dans le poumon asthmatique, l'expression de CX3CLl est également fortement augmentée au niveau des cellules musculaires lisses. Dans notre modèle murin d'asthme induit par l'antigène majeur du parasite Leishmania, des lymphocytes T $C D 4^{+}$, spécifiques de l'antigène, infiltrent la musculature lisse (données non publiées).

\section{Voies de signalisation initiées par le récepteur CX3CR1}

La fixation des chimokines à leur(s) récepteur(s) active des voies de transduction du signal contrôlant la réorganisation du cytosquelette et l'activation des intégrines qui conduit à une augmentation de l'adhérence et de la migration cellulaire. La seule fixation de CX3CLI à son récepteur $\mathrm{CX} 3 \mathrm{CR} 1$ conduit à une stimulation du calcium intracellulaire, des voies des mitogen-activated protein kinase (MAPK) et de la phosphoinositide 3-kinase (PI3K) et de Akt/PKB (protéine kinase B) [40, 41] qui régulent de nombreux gènes en rapport avec la survie et l'apoptose. Les voies de signalisation induites après l'activation par CX3CRl ont été étudiées dans les neurones [42] et les cellules microgliales de rat [40], les cellules $\mathrm{CHO}^{8}$ transfectées et exprimant $\mathrm{CX} 3 \mathrm{CR} 1$ humain [43] et dans de nombreuses lignées monocytaires humaines [41]. Contrairement au récepteur humain, le CX3CRI murin, exprimé dans des cellules CHO, n'active pas les voies de signalisation dépendantes de la PI3K [44]. Ceci serait dû à la présence, en position 326 dans l'extrémité carboxy-terminale de la protéine murine, d'un résidu proline remplaçant la sérine de la protéine humaine. Dans la microglie, l'activation d'Akt conduit à la phosphorylation de la protéine proapoptotique BAD. CX3CLI inhibe l'expression des gènes antiapoptotiques $B I D$ et $B A X$ de même que l'expression de $\mathrm{BCL}-\mathrm{XL}$ ( $B$-cell lymphoma-extra large) $[37,39]$. Dans les cellules HEK2939 transfectées et exprimant CX3CR1, CX3CL1 induit un flux calcique [45]. Les voies d'activation dépendant de la MAPK ERK (extracellular signal-regulated kinase) et d'Akt restent à explorer.

\section{Applications thérapeutiques}

Bien que les corticostéroïdes inhalés soient des antiinflammatoires très efficaces pour le traitement de l'asthme persistant, ils ne guérissent pas la maladie et $10 \%$ des patients asthmatiques ne répondent pas à ce type de traitement [46]. Des thérapies plus ciblées, comme des anticorps spécifiques de l'IL-5, ont été testées mais, sur des populations de patients asthmatiques sans sélection de sous-populations sur base de critères particuliers, les résultats se sont avérés plutôt décevants [47]. Les corticostéroïdes et les bronchodilatateurs $\beta 2$-agonistes ${ }^{10}$ représentent le traitement standard de l'asthme. Nos résultats, montrant que l'administration locale d'un antagoniste de CX3CLl à des animaux sensibilisés à un allergène conduit à une diminution de l'inflammation des voies aériennes et de l'hyperréactivité bronchique, apparaissent donc très prometteurs. Ces travaux devraient inciter le lancement

${ }^{8}$ Les cellules $\mathrm{CHO}$ sont dérivées de l'ovaire du hamster chinois. Elles sont couramment utilisées pour les expériences de transfection (apport d'un gène exogène afin d'étudier son expression et les propriétés de la protéine correspondante).

${ }^{9}$ Comme les cellules CHO, les cellules humaines HEK293 (human embryonic kidney 293 cells) sont utilisées pour les expériences de transfection.

${ }^{10}$ Les $\beta 2$-agonistes diminuent la neurotransmission cholinergique, et donc amplifient l'effet bronchodilatateur des anticholinergiques. 
d'études cliniques afin d'explorer les effets de tels antagonistes de CX3CRI dans l'asthme humain. Les effets spectaculaires du traitement par voie topique de la dermatite atopique par l'antagoniste de CX3CL1, qui a donné lieu à un brevet, représentent également une piste pour de futurs développements.

\section{Conclusions}

Les chimiokines ont été initialement identifiées comme des cytokines de faible masse moléculaire qui induisaient une migration directionnelle in vitro. Depuis, d'autres rôles de ces chimiokines ont été identifiés dans la survie cellulaire, l'angiogenèse, la réparation cellulaire, la progression tumorale, l'hématopoïèse, l'immunité antimicrobienne et l'analgésie. CX3CR1, dont l'expression est augmentée dans les pathologies allergiques, joue un rôle majeur dans le développement de l'asthme, indépendamment du chimiotactisme, en favorisant la survie des lymphocytes Th2 dans le poumon inflammatoire et, dans la dermatite atopique, en favorisant la rétention des lymphocytes Thl et Th2. L'identification de ces nouvelles capacités fonctionnelles permet non seulement de préciser les mécanismes conduisant aux pathologies allergiques mais également de mieux comprendre les conséquences des déficiences en chimiokines ou en leurs récepteurs. $\diamond$

\section{SUMMARY}

\section{Role of fractalkine/CX3CL1 and its receptor CX3CR 1 in allergic diseases}

Allergic asthma and atopic dermatitis are diseases mainly resulting from the activation of Th2 cells, that produce cytokines favouring lg $\varepsilon$ production and eosinophilia but also of Thl cells, that contribute to inflammation chronicity. Lymphocyte recruitment and retention of Th cells in target organs are 2 key events for asthma and atopic dermatitis pathogenesis. While lymphocyte migration is regulated by chemokines and lipid mediators such as leukotrienes and prostaglandins, factors involved in lymphocyte retention and survival within inflammatory tissues remain poorly understood. Recent works show that, in allergic diseases, there is an increased expression of fractalkine/CX3CLI and its unique receptor $C X 3 C R I$ and that this chemokine does not act as chemoattractant. In allergic asthma, CX3CRI expression regulates Th2 and Thl cell survival in the inflammatory lung, while, in atopic dermatitis, it regulate Th2 and Thl cell retention into the inflammatory site. Use of peptides blocking fractalkine binding to its receptor is currently tested in the treatment of asthma and atopic dermatitis. $\diamond$

\section{REMERCIEMENTS}

Les résultats repris dans ce travail résultent en partie de financements de la Fondation pour la recherche médicale (Nouveaux défis en allergologie), de l'Agence nationale de la recherche, ANR-06-MIME-008, E.G.I.D. ANR-10-LABX-46, de l'Union européenne et d'AVIESAN (ITMO IHP).

\section{LIENS D'INTÉRÊT}

Les auteurs déclarent n'avoir aucun lien d'intérêt concernant les données publiées dans cet article.

\section{RéFÉRENCES}

1. Maziak W, Behrens T, Brasky TM, et al. Are asthma and allergies in children and adolescents increasing? Results from ISAAC phase I and phase III surveys in Munster, Germany. Allergy 2003 ; 58 : 572-9.

2. Saito H. Much atopy about the skin: genome-wide molecular analysis of atopic eczema. Int Arch Allergy Immunol 2005 ; 137 : 319-25.

3. Spergel JM. From atopic dermatitis to asthma: the atopic march. Ann Allergy Asthma Immunol 2010 ; 105 : 99-106; quiz 7-9, 17.

4. Wills-Karp M. Immunologic basis of antigen-induced airway hyperresponsiveness. Annu Rev Immunol 1999 ; 17 : 255-81.

5. Masoli M, Fabian D, Holt S, Beasley R. The global burden of asthma: executive summary of the GINA Dissemination Committee report. Allergy $2004 ; 59: 469-78$.

6. Bazan JF, Bacon KB, Hardiman G, et al. A new class of membrane-bound chemokine with a CX3C motif. Nature 1997 ; $385: 640-4$.

7. Booth BW, Sandifer T, Martin EL, Martin LD. IL-13-induced proliferation of airway epithelial cells: mediation by intracellular growth factor mobilization and ADAM17. Respir Res $2007 ; 8: 51$.

8. Hartl D, He CH, Koller B, et al. Acidic mammalian chitinase is secreted via an ADAM17/epidermal growth factor receptor-dependent pathway and stimulates chemokine production by pulmonary epithelial cells. J Biol Chem $2008 ; 283: 33472-82$.

9. Nakayama T, Watanabe $Y$, Oiso N, et al. Eotaxin-3/CC chemokine ligand 26 is a functional ligand for CX3CR1. J Immunol 2010 ; 185 : 6472-9.

10. Schwaeble WJ, Stover CM, Schall TJ, et al. Neuronal expression of fractalkine in the presence and absence of inflammation. FEBS Lett 1998 ; 439 : 203-7.

11. Lucas AD, Chadwick N, Warren BF, et al. The transmembrane form of the $\mathrm{CX} 3 \mathrm{CLl}$ chemokine fractalkine is expressed predominantly by epithelial cells in vivo. Am J Pathol $2001 ; 158: 855-66$

12. Rimaniol AC, Till SJ, Garcia G, et al. The CX3C chemokine fractalkine in allergic asthma and rhinitis. J Allergy Clin Immunol 2003 ; 112 : 1139-46.

13. Echigo T, Hasegawa M, Shimada Y, et al. Expression of fractalkine and its receptor, CX3CR1, in atopic dermatitis: possible contribution to skin inflammation. J Allergy Clin Immunol $2004 ; 113$ : 940-8.

14. El-Shazly A, Berger P, Girodet PO, et al. Fraktalkine produced by airway smooth muscle cells contributes to mast cell recruitment in asthma. J Immunol $2006 ; 176: 1860-8$.

15. Kim KW, Vallon-Eberhard A, Zigmond $\varepsilon$, et al. In vivo structure/function and expression analysis of the $\mathrm{CX} 3 \mathrm{C}$ chemokine fractalkine. Blood $2011 ; 118$ : el56-67.

16. Harrison JK, Jiang $Y$, Chen $S$, et al. Role for neuronally derived fractalkine in mediating interactions between neurons and CX3CR1-expressing microglia. Proc Natl Acad Sci USA 1998 ; 95 : 10896-901.

17. Imai T, Hieshima K, Haskell C, et al. Identification and molecular characterization of fractalkine receptor CX3CR1, which mediates both leukocyte migration and adhesion. Cell $1997 ; 91: 521-30$.

18. Jung $S$, Aliberti J, Graemmel P, et al. Analysis of fractalkine receptor CX(3) CRl function by targeted deletion and green fluorescent protein reporter gene insertion. Mol Cell Biol $2000 ; 20: 4106-14$.

19. Chang SY, Song JH, Guleng B, et al. Circulatory antigen processing by mucosal dendritic cells controls CD8 ${ }^{+}$T cell activation. Immunity $2013 ; 38$ : 153-65.

20. Liu ZQ, Wu Y, Song JP, et al. Tolerogenic CX3CRI+ B cells suppress food allergy-induced intestinal inflammation in mice. Allergy 2013; $68: 1241-8$.

21. Fraticelli P, Sironi $M$, Bianchi $G$, et al. Fractalkine (CX3CL1) as an amplification circuit of polarized Thl responses. J Clin Invest 2001; 107 : 1173-81.

22. Ning W, Li CJ, Kaminski N, et al. Comprehensive gene expression profiles reveal pathways related to the pathogenesis of chronic obstructive pulmonary disease. Proc Natl Acad Sci USA 2004 ; 101 : 14895-900.

23. Balabanian $K$, Foussat $A$, Dorfmuller $P$, et al. $C X(3) C$ chemokine fractalkine in pulmonary arterial hypertension. Am J Respir Crit Care Med 2002; 165 : 1419-25.

24. Marasini B, Cossutta R, Selmi C, et al. Polymorphism of the fractalkine receptor $C X 3 C R 1$ and systemic sclerosis-associated pulmonary arterial hypertension. Clin Dev Immunol 2005 ; 12 : 275-9.

25. Murphy G, Caplice N, Molloy M. Fractalkine in rheumatoid arthritis: a review to date. Rheumatology (Oxford) $2008 ; 47: 1446-51$.

26. Lesnik P, Haskell CA, Charo IF. Decreased atherosclerosis in $C X 3 C R 1^{-/-}$mice reveals a role for fractalkine in atherogenesis. J Clin Invest 2003; 111 : $333-40$. 


\section{RÉFÉRENCES}

27. Tremblay K, Lemire $M$, Provost $V$, et al. Association study between the $C X 3 C R 1$ gene and asthma. Genes Immun $2006 ; 7: 632-9$.

28. Depner M, Kormann MS, Klopp N, et al. CX3CR1 Polymorphisms are associated with atopy but not asthma in German children. Int Arch Allergy Immunol 2007 ; 144 : $91-4$.

29. Mionnet $C$, Buatois $V$, Kanda $A$, et al. CX3CRl is required for airway inflammation by promoting $T$ helper cell survival and maintenance in inflamed lung. Nat Med $2010 ; 16: 1305-12$.

30. Staumont-Salle D, Fleury S, Lazzari A, et al. CX3CL1 (fractalkine) and its receptor CX3CR1 regulate atopic dermatitis by controlling effector T cell retention in inflamed skin. J Exp Med $2014 ; 211$ : $1185-96$.

31. Nishimura M, Umehara $H$, Nakayama T, et al. Dual functions of fractalkine/CX3C ligand 1 in trafficking of perforin ${ }^{+}$/granzyme $B^{+}$cytotoxic effector lymphocytes that are defined by CX3CRI expression. J Immunol $2002 ; 168: 6173-80$.

32. Garin $A$, Pellet $P$, Deterre $P$, et al. Cloning and functional characterization of the human fractalkine receptor promoter regions. Biochem J 2002 ; 368 : 753-60.

33. Ramos MV, Fernandez GC, Brando RJ, et al. Interleukin-10 and interferon-gamma modulate surface expression of fractalkine-receptor (CX(3)CRI) via PI3K in monocytes. Immunology 2010 ; $129: 600-9$.

34. Sechler JM, Barlic J, Grivel JC, Murphy PM. IL-15 alters expression and function of the chemokine receptor CX3CR1 in human NK cells. Cell Immunol $2004 ; 230$ : 99-108.

35. Meucci 0 , Fatatis A, Simen AA, Miller RJ. Expression of CX3CR1 chemokine receptors on neurons and their role in neuronal survival. Proc Natl Acad Sci USA $2000 ; 97: 8075-80$.

36. Tong N, Perry SW, Zhang $Q$, et al. Neuronal fractalkine expression in HIV-l encephalitis: roles for macrophage recruitment and neuroprotection in the central nervous system. J Immunol 2000 ; $164: 1333-9$.

37. Boehme SA, Lio FM, Maciejewski-Lenoir D, et al. The chemokine fractalkine inhibits Fas-mediated cell death of brain microglia. J Immunol 2000 ; 165 : 397-403.

38. Mizuno T, Kawanokuchi J, Numata K, Suzumura A. Production and neuroprotective functions of fractalkine in the central nervous system. Brain Res $2003 ; 979: 65-70$.

39. Landsman L, Bar-On L, Zernecke A, et al. CX3CRl is required for monocyte homeostasis and atherogenesis by promoting cell survival. Blood $2009 ; 113: 963-72$.
40. Maciejewski-Lenoir D, Chen S, Feng L, et al. Characterization of fractalkine in rat brain cells: migratory and activation signals for CX3CR-1-expressing microglia. J Immunol 1999 ; 163 : 1628-35.

41. Cambien B, Pomeranz M, Schmid-Antomarchi H, et al. Signal transduction pathways involved in soluble fractalkine-induced monocytic cell adhesion. Blood $2001 ; 97: 2031-7$

42. Meucci 0 , Fatatis A, Simen AA, et al. Chemokines regulate hippocampal neuronal signaling and gp120 neurotoxicity. Proc Natl Acad Sci USA 1998 ; 95 : 14500-5.

43. Kansra V, Groves C, Gutierrez-Ramos JC, Polakiewicz RD. Phosphatidylinositol 3-kinase-dependent extracellular calcium influx is essential for CX(3) CRI-mediated activation of the mitogen-activated protein kinase cascade. J Biol Chem $2001 ; 276: 31831-8$.

44. Davis $\mathrm{CN}$, Harrison JK. Proline 326 in the $\mathrm{C}$ terminus of murine $\mathrm{CX} 3 \mathrm{CR}$ prevents $\mathrm{G}$-protein and phosphatidylinositol 3-kinase-dependent stimulation of Akt and extracellular signal-regulated kinase in Chinese hamster ovary cells. J Pharmacol Exp Ther 2006 ; 316: 356-63.

45. Combadiere C, Gao J, Tiffany HL, Murphy PM. Gene cloning, RNA distribution, and functional expression of $\mathrm{mCX} 3 \mathrm{CRl}$, a mouse chemotactic receptor for the CX3C chemokine fractalkine. Biochem Biophys Res Commun $1998 ; 253$ : 728-32.

46. Lopez Vina A. Severe asthma refractory to treatment: concepts and realities. Arch Bronconeumol $2006 ; 42: 20-5$.

47. O'Byrne PM. The demise of anti IL-5 for asthma, or not. Am J Respir Crit Care Med $2007 ; 176: 1059-60$

48. Froidure $A$, Charles P. Les cellules dendritiques humaines dans l'asthme et la rhinite allergique. Med Sci (Paris) $2015 ; 31: 151-8$

49. De Masson A, Bouaziz JD, Battistella M, et al. Immunopathologie du psoriasis : from bench to bedside. Med Sci (Paris) 2016 ; 32 : 253-9.

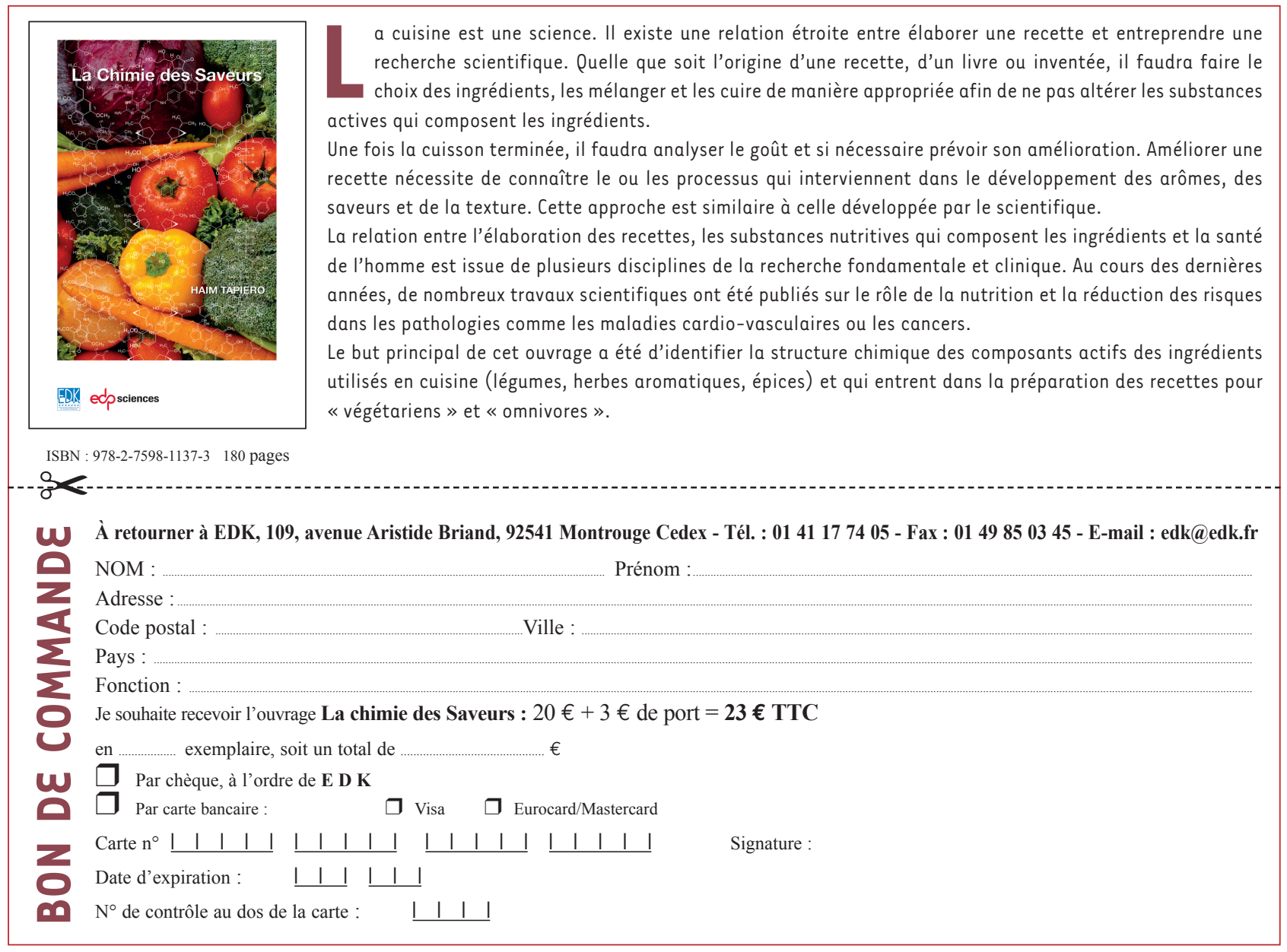

